\title{
Correlation of imaging and histopathology of thrombi in acute ischemic stroke with etiology and outcome: a systematic review
}

\author{
Waleed Brinjikji, ${ }^{1}$ Sharon Duffy, ${ }^{2}$ Anthony Burrows, ${ }^{1}$ Werner Hacke, ${ }^{3}$ \\ David Liebeskind, ${ }^{4}$ Charles B L M Majoie, ${ }^{5}$ Diederik W J Dippel, ${ }^{6}$ Adnan H Siddiqui, ${ }^{7}$ \\ Pooja Khatri, ${ }^{8}$ Blaise Baxter, ${ }^{9}$ Raul Nogeuira, ${ }^{10}$ Matt Gounis, ${ }^{11}$ Tudor Jovin, ${ }^{12}$ \\ David F Kallmes ${ }^{1}$
}

For numbered affiliations see end of article.

\section{Correspondence to} Dr Waleed Brinjikji, Department of Radiology, Mayo Clinic, 200 1st Street SW, Rochester, MN 55905, USA:

Brinjikji.waleed@mayo.edu

Received 12 March 2016 Revised 15 April 2016 Accepted 22 April 2016 Published Online First 10 May 2016

\section{CrossMark}

To cite: Brinjikji W, Duffy S, Burrows A, et al. J Neurolntervent Surg 2017;9:529-534.

\section{ABSTRACT}

Background and purpose Studying the imaging and histopathologic characteristics of thrombi in ischemic stroke could provide insights into stroke etiology and ideal treatment strategies. We conducted a systematic review of imaging and histologic characteristics of thrombi in acute ischemic stroke.

Materials and methods We identified all studies published between January 2005 and December 2015 that reported findings related to histologic and/or imaging characteristics of thrombi in acute ischemic stroke secondary to large vessel occlusion. The five outcomes examined in this study were (1) association between histologic composition of thrombi and stroke etiology; (2) association between histologic composition of thrombi and angiographic outcomes; (3) association between thrombi imaging and histologic characteristics; (4) association between thrombi imaging characteristics and angiographic outcomes; and (5) association between imaging characteristics of thrombi and stroke etiology. A meta-analysis was performed using a random effects model.

Results There was no significant difference in the proportion of red blood cell (RBC)-rich thrombi between cardioembolic and large artery atherosclerosis etiologies (OR 1.62, 95\% Cl 0.1 to 28.0, $p=0.63$ ). Patients with a hyperdense artery sign had a higher odds of having RBC-rich thrombi than those without a hyperdense artery sign (OR 9.0, 95\% Cl 2.6 to 31.2, $\mathrm{p}<0.01$ ). Patients with a good angiographic outcome had a mean thrombus Hounsfield unit (HU) of 55.1 \pm 3.1 compared with a mean $\mathrm{HU}$ of $48.4 \pm 1.9$ for patients with a poor angiographic outcome (mean standard difference 6.5, $95 \% \mathrm{Cl} 2.7$ to $10.2, \mathrm{p}<0.001)$. There was no association between imaging characteristics and stroke etiology (OR 1.13, 95\% Cl 0.32 to $4.00, p=0.85$ ).

Conclusions The hyperdense artery sign is associated with RBC-rich thrombi and improved recanalization rates. However, there was no association between the histopathological characteristics of thrombi and stroke etiology and angiographic outcomes.

\section{INTRODUCTION}

Prior studies have suggested that studying thrombus composition can provide insights into stroke etiology, predict recanalization success following both intravenous thrombolysis and mechanical thrombectomy, and assist in the development of new technologies aimed at retrieving specific types of thrombi. ${ }^{1-6}$ In fact, recently published in vitro studies have suggested that clot composition can affect the ideal choice of techniques used during clot retriever and does, in fact, play a role in device/clot interaction. ${ }^{7} 8$ Because stent-retriever and aspiration thrombectomy technologies which allow us to retrieve and study fresh thrombi in patients with acute stroke have only recently been developed, the current body of literature on the association between imaging and histopathological characteristics of clot and their association with recanalization outcomes and stroke etiology is primarily limited to small single-center case series. Because of this, it is difficult to make any definitive conclusions regarding the importance of imaging and histopathological characteristics of thrombi and outcomes and etiology of acute stroke.

In order to update the neurointerventional community on the current status of thrombus research in acute ischemic stroke secondary to large vessel occlusion, we conducted a systematic review and meta-analysis of research on thrombi in acute ischemic stroke aimed at studying the following five outcomes: (1) the association between histologic composition of thrombi and stroke etiology; (2) the association between thrombus histologic composition and angiographic outcomes; (3) the association between imaging and histologic characteristics of thrombi in stroke; (4) the association between imaging characteristics of thrombi and angiographic outcomes; and (5) the association between imaging characteristics of thrombi and stroke etiology.

\section{MATERIALS AND METHODS}

\section{Literature search}

We identified all studies published between January 2005 and December 2015 that reported findings related to the histologic and/or imaging characteristics of thrombi in patients with acute ischemic stroke secondary to large vessel occlusion. A comprehensive literature search of the databases PubMed, Ovid MEDLINE and Ovid EMBASE was designed and conducted by a librarian with 30 years of experience in systematic reviews and meta-analysis with input from the authors. The keywords clot, thrombus, embolus, stroke, ischemic 
stroke, large vessel occlusion, mechanical thrombectomy, CT, MRI, pathology and histology were used in both 'AND' and 'OR' combinations. Studies were selected using the following inclusion criteria: (1) patients with large vessel occlusion; (2) either imaging or histologic characteristics of clots in acute stroke patients were reported; (3) the study examined at least one of the five outcomes listed above; (4) retrospective or prospective articles or conference abstracts with at least five patients; and (5) published in English or an English translation was available. Abstracts had to clearly report results such as number of patients, means and SDs for continuous variables, proportions for continuous variables or provide content in table form. Large vessel occlusion was defined as occlusion of the vertebral, basilar carotid terminus, middle and anterior cerebral arteries as determined by MR angiography, CT angiography or digital subtraction angiography. Exclusion criteria were the following: (1) studies which did not report large vessel occlusion patients or did not stratify results by large and small vessel occlusion; (2) studies which did not report imaging or histologic characteristics of stroke, or reported outcomes in the subacute phase; (3) autopsy studies; and (4) studies with $<5$ patients. For the systematic review and meta-analysis examining the association of imaging characteristics of thrombi and angiographic outcomes, studies which only reported outcomes of intravenous tissue plasminogen activator (IV-tPA) were excluded.

Two authors independently searched the database and selected potentially relevant articles based on the title and abstract and obtained the full text for detailed review. We searched the reference lists of retrieved articles and published review articles for additional studies. We also screened duplicate publications that drew on the same datasets (ie, data overlapped with that in other included studies). In cases of duplication or overlapping patient populations, only the publication with the most complete data was included. Data overlap was determined if the patients included in two different studies were from the same institution and overlapping time periods. The most complete set of data was defined as the set of data with the largest number of patients or most amount of detail in terms of results reporting. We contacted authors of some studies to request incomplete or unpublished data.

\section{Outcomes}

We studied five different outcomes in this study. First, we studied the association between the histologic composition of thrombi (ie, red blood cell (RBC) content and fibrin content) and stroke etiology. Stroke etiology was generally defined as large artery atherosclerosis or cardioembolic. The second outcome studied was the association between the histologic composition of thrombi and recanalization outcomes. For this, histologic composition was defined by the RBC and fibrin content. Recanalization outcomes were defined as good if the Thrombolysis In Myocardial Infarction (TIMI) score was $\geq 2$ or if the Thrombolysis In Cerebral Infarction (TICI) score was $\geq 2 b$. For the third outcome we studied the association between the imaging and histologic characteristics of thrombi in stroke. For this, both CT and MRI were used. CT outcomes were evaluated using Hounsfield unit (HU) measurements and MRI characteristics were defined using the presence or absence of the susceptibility vessel sign (SVS) (ie, low signal on susceptibility-weighted imaging (SWI)). Histologic characteristics were analyzed by RBC and fibrin content. Fourth, we studied the association between imaging characteristics (on MRI and CT) of thrombi and angiographic outcomes. Again, for this, good angiographic outcome was defined as a TIMI score of $\geq 2$ or TICI score of $\geq 2 \mathrm{~b}$. For this analysis we also performed a subgroup analysis on the association between HU and outcomes by including only those articles which reported the use of techniques such as aspiration thrombectomy and stent-retrievers. Lastly, we studied the association between imaging characteristics of thrombi on CT and MRI (ie, HUs and SWI characteristics) and stroke etiology (cardioembolic and large artery atherosclerosis).

\section{Histologic and imaging characteristics}

The histologic characteristics of thrombi studied included the proportion of RBCs, white blood cells (WBCs), and fibrin in retrieved thrombi. In some cases we were able to study the histologic composition as a categorical variable (ie, RBC-rich, RBC-poor, fibrin-rich, fibrin-poor, etc); however, in many cases the studies only reported the mean proportion of RBCs, WBCs, and fibrin between groups. In cases where the association between stroke etiology and histologic and imaging characteristics of thrombi were studied, the etiology was categorized as large artery atherosclerosis or cardioembolic.

Data from included studies were extracted independently by two authors using a standardized form and any disagreement was resolved by consensus. Histologic analysis was generally performed using H\&E staining. Imaging characteristics of thrombi studied included the presence or absence of a hyperdense artery sign (HAS) and/or thrombi HUs on non-contrast CT or the presence/absence of SVS on MRI at the time of presentation. Details of the staining and imaging methods used in each study are provided in table 1.

\section{Statistical analysis}

All included studies were comparative. Meta-analysis results were expressed as an OR for binary outcomes using random effects meta-analysis. ${ }^{9}$ Random effects meta-analysis was also used to study continuous outcomes with outcomes reported using the mean standard difference (MSD). For all outcomes, we quantified between-study heterogeneity using homogeneity by calculating the $\mathrm{I}^{2}$ statistics. ${ }^{10} \mathrm{~A}$ formal statistical meta-analysis was used whenever there were at least three studies examining any given outcome. In cases where data from studies were not presented in an easily extractable manner or fewer than three studies examined a specific outcome, data were summarized in the form of a systematic review.

\section{Risk of bias assessment}

Risk of bias assessment was performed using the NewcastleOttawa Scale. This tool is used for assessing the risk of bias of non-randomized studies included in systematic reviews or meta-analyses. Each study is judged on eight items categorized into three groups: (1) selection of the study groups; (2) comparability of the study groups; and (3) ascertainment of the outcome of interest. ${ }^{11}$

\section{RESULTS}

\section{Literature search and risk of bias assessment}

The initial literature search yielded a total of 338 studies. Of these, 297 were excluded based on title and abstract alone. Sixteen additional articles were excluded due to insufficient available data. In total, 25 articles were included in the final systematic review and meta-analysis. All studies were retrospective and all studies were at high risk of bias using the NewcastleOttawa criteria. Nine articles including 322 patients examined associations between histology and etiology, four articles including 158 patients examined associations between thrombi histopathology and outcome, eight articles including 250 patients 
Table 1 Summary of included studies

\begin{tabular}{|c|c|c|c|c|c|}
\hline Outcome studied & Author, year & No of patients & Therapy type & Histological techniques & $\begin{array}{l}\text { Imaging } \\
\text { techniques }\end{array}$ \\
\hline \multirow{9}{*}{ Histology and etiology } & Simons, 2015 & 40 & MT & $\mathrm{H} \& \mathrm{E}$ and $\mathrm{CD} 34$ & $\mathrm{CT}$ \\
\hline & Kim, 2015 & 37 & MT & H\&E, PGPIIIa, CD61 & MRI \\
\hline & Singh, 2014 & 49 & MT & $\mathrm{H} \& \mathrm{E}$ & NA \\
\hline & Niesten, 2014 & 22 & MT & $\begin{array}{l}\text { H\&E, Mallory's phosphotungistic } \\
\text { acid-hematoxilin, glyophorin A, CD31 }\end{array}$ & $\mathrm{CT}$ \\
\hline & Boekh-Behrens, 2014 & 54 & MT & $\mathrm{H} \& \mathrm{E}$, Elastica van Gieson & $\mathrm{CT}$ \\
\hline & Sallustio, 2014 & 28 & MT & $\mathrm{H} \& \mathrm{E}$ & NA \\
\hline & Sato, 2012 & 17 & None & H\&E, PGPIIb/IIla, glycophorin A and Fibll & NA \\
\hline & Liebeskind, 2011 & 50 & MT & $H \& E$ & $\mathrm{CT} / \mathrm{MRI}$ \\
\hline & Marder, 2006 & 25 & MT & $\mathrm{H} \& \mathrm{E}$ & NA \\
\hline \multirow{4}{*}{$\begin{array}{l}\text { Histology and angiographic } \\
\text { outcomes }\end{array}$} & Boekh-Behrens, 2014 & 54 & MT & H\&E, Elastica van Gieson & $\mathrm{CT}$ \\
\hline & Liebeskind, 2011 & 50 & MT & $H \& E$ & CT/MRI \\
\hline & Marder, 2006 & 25 & MT & $\mathrm{H} \& \mathrm{E}$ & NA \\
\hline & Qureshi, 2015 & 19 & MT & $H \& E$ & NA \\
\hline \multirow[t]{8}{*}{ Imaging and histology } & Simons, 2015 & 40 & MT & $\mathrm{H} \& \mathrm{E}$ and $\mathrm{CD} 34$ & $\mathrm{CT}$ \\
\hline & Kim, 2015 & 37 & MT & H\&E, PGPIIIa, CD61 & MRI \\
\hline & Niesten, 2014 & 22 & MT & $\begin{array}{l}\text { H\&E, Mallory's phosphotungistic } \\
\text { acid-hematoxilin, glyophorin A, CD31 }\end{array}$ & $\mathrm{CT}$ \\
\hline & Liebeskind, 2011 & 50 & MT & $H \& E$ & $\mathrm{CT} / \mathrm{MRI}$ \\
\hline & Cline, 2013 & 16 & MT & $\mathrm{H} \& \mathrm{E}$ & $\mathrm{CT}$ \\
\hline & Lopez-Cancio, 2013 & 15 & MT & Fibrinogen, PAI-1, P-selectin, VWf & $\mathrm{CT}$ \\
\hline & Boekh-Behrens, 2014 & 54 & MT & H\&E, Elastica van Gieson & $\mathrm{CT}$ \\
\hline & Ahn, 2012 & 16 & MT & $\mathrm{H} \& \mathrm{E}$ & $\mathrm{CT}$ \\
\hline \multirow{8}{*}{$\begin{array}{l}\text { Imaging and outcome of IA } \\
\text { therapy }\end{array}$} & Moftakher, 2013 & 77 & MT & NA & $\mathrm{CT}$ \\
\hline & Kim, 2006 & 34 & IA tPA & NA & $\mathrm{CT}$ \\
\hline & Mokin, 2015 & 41 & MT & NA & $\mathrm{CT}$ \\
\hline & Soize, 2015 & 113 & MT & NA & MRI \\
\hline & Liebeskind, 2011 & 50 & MT & $\mathrm{H} \& \mathrm{E}$ & CT/MRI \\
\hline & Froehler, 2013 & 67 & MT & NA & $\mathrm{CT}$ \\
\hline & Spiotta, 2014 & 95 & MT & NA & $\mathrm{CT}$ \\
\hline & Vijiaratnam, 2015 & 181 & MT & NA & $\mathrm{CT}$ \\
\hline \multirow[t]{7}{*}{ Imaging and etiology } & Topcuoglu, 2015 & 105 & IV-tPA or IA-tPA & NA & $\mathrm{CT}$ \\
\hline & Froehler, 2013 & 67 & MT & NA & $\mathrm{CT}$ \\
\hline & Puig, 2012 & 45 & IV-tPA & NA & $\mathrm{CT}$ \\
\hline & Kim, 2006 & 34 & IA/IV-tPA & NA & $\mathrm{CT}$ \\
\hline & Almekhlafi, 2011 & 264 & IA/IV & NA & CT/MRI \\
\hline & Guthrie, 2012 & 128 & NA & NA & $\mathrm{CT}$ \\
\hline & Cho, 2005 & 95 & NA & NA & MRI \\
\hline
\end{tabular}

IA, intra-arterial; IV, intravenous; MT, mechanical thrombectomy; NA, not applicable; tPA, tissue plasminogen activator.

examined associations between histology and imaging characteristics, eight articles with 658 patients examined associations between imaging and revascularization with intra-arterial therapy, and seven articles with 738 patients examined the association between imaging and etiology. A summary of the included studies is provided in table 1 and figure 1 summarizes the search strategy.

\section{Histologic composition of thrombi and stroke etiology}

Nine articles including 322 patients examined the association between thrombi histology and etiology. ${ }^{5}{ }^{12-19}$ Of these nine studies, five $(55.5 \%)$ found no association between thrombi histology and etiology. Two studies (22.2\%) found that cardioembolic strokes were associated with RBC-rich thrombi, one study (11.1\%) found that large artery atherosclerosis was associated with RBC-rich thrombi, and one study (11.1\%) found that cardioembolic strokes were associated with WBC-rich thrombi.

Four studies had data available for extraction for purposes of a meta-analysis. There was no significant difference in the proportion of RBC-rich thrombi between cardioembolic and large artery atherosclerosis etiologies (OR 1.62, 95\% CI 0.1 to 28.0, $\mathrm{p}=0.63)$. The mean RBC composition for cardioembolic strokes was $39.3 \%$ and $29.5 \%$ for large artery atherosclerosis (MSD 9.8, $95 \%$ CI -15.9 to $35.5, p=0.45)$. The mean fibrin composition for cardioembolic strokes was $28.4 \%$ and $40.0 \%$ for large artery atherosclerosis (MSD -11.6 , 95\% CI -41.8 to $18.7, \mathrm{p}=0.46) . \mathrm{I}^{2}$ values were $>50 \%$ for all outcomes, indicating substantial heterogeneity.

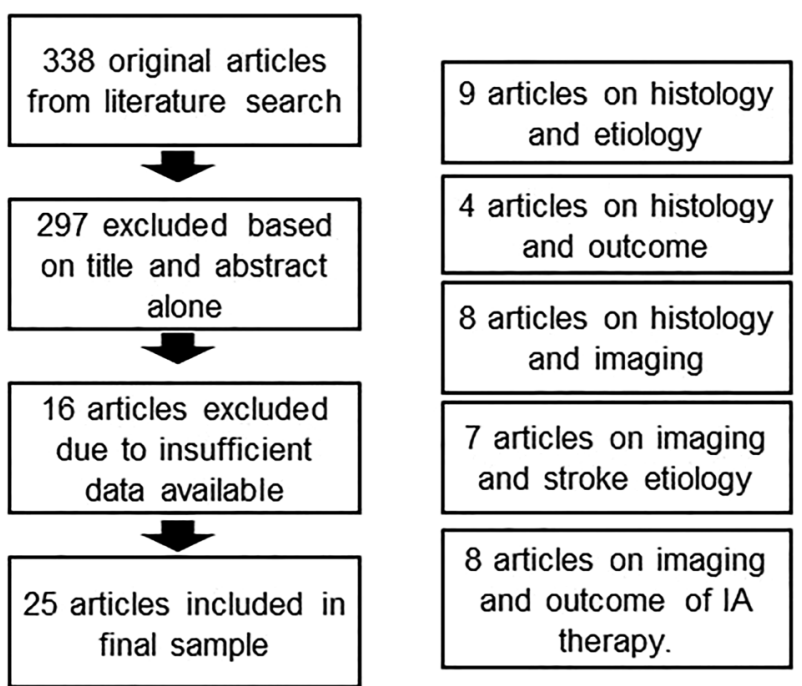

Figure 1 Search strategy results. 


\section{Clot histologic composition and angiographic outcomes}

Four articles with 158 patients examined the association between thrombi histology and angiographic outcomes. ${ }^{5} 121420$ In three studies $(75.0 \%)$ there was no association between thrombi histology and outcome and in one study WBC-rich thrombi were associated with less favorable recanalization rates. No meta-analysis for this outcome could be performed.

\section{Imaging and histologic characteristics of thrombi in stroke}

Eight articles including 250 patients examined associations between thrombi histology and imaging characteristics. $^{1} 5121315182122$ Six of six articles (100\%, 6/6) examining the association between RBC and fibrin content and HAS demonstrated that hyperdense thrombus indicated a high RBC content. Two of two articles $(100 \%, 2 / 2)$ examining the association between $\mathrm{RBC}$ and fibrin content found that the presence of the SVS indicated the presence of RBC-rich thrombi.

Three articles had data available for extraction for purposes of a meta-analysis. HAS was associated with RBC-rich thrombi. Patients with HAS had a higher odds of having RBC-rich thrombi than those without a HAS on CT (OR 9.0, 95\% CI 2.6 to $31.2, \mathrm{p}<0.01)$. $\mathrm{I}^{2}$ for this estimate was $0 \%$, indicating no substantial heterogeneity in the analysis of this outcome. The mean RBC\% for patients with a HAS was $45.2 \%$ compared with 23.3\% for those without a HAS (MSD 24.5, 95\% CI 17.4 to $31.7, \mathrm{p}<0.01)$. $\mathrm{I}^{2}$ for this estimate was $>50 \%$, indicating substantial heterogeneity (figure 2).

\section{Imaging characteristics of thrombi and angiographic outcomes}

A total of eight studies with 658 patients examined the association between thrombi imaging characteristics and angiographic outcomes following mechanical thrombectomy. ${ }^{3}{ }^{52-32}$ As mentioned in the Methods section, IV-tPA studies were excluded from this analysis. Seven studies examined outcomes with mechanical thrombectomy with or without IA-tPA and one study examined outcomes with IA-tPA alone. Five studies used modern techniques including stent-retrievers and aspiration thrombectomy, two studies reported outcomes of Merci clot retrieval alone, and one study included patients receiving IA-tPA alone. Of the eight studies, six examined the correlation between clot density on CT and outcomes, one examined the correlation between clot signal on MRI and outcomes, and one study examined the correlation between clot characteristics on both CT and MRI and outcomes.

Of the studies examining the association between CT attenuation of thrombi and outcomes, five $(71.4 \%, 5 / 7)$ found that the presence of a HAS was associated with successful recanalization, one study $(14.3 \%, 1 / 7)$ found no association between the HAS and recanalization, and one study $(14.3 \%, 1 / 7)$ found a negative association between the HAS and recanalization. Of the two studies examining recanalization rates with MRI, both $(100 \%$, $2 / 2$ ) found a positive association between the SVS and recanalization.

Five articles had data available for meta-analysis on the correlation with thrombi density on CT and angiographic outcomes. Patients with a good angiographic outcome had a mean HU of $55.1 \pm 3.1$ compared with a mean HU of $48.4 \pm 1.9$ for patients with a poor angiographic outcome (MSD 6.5, 95\% CI 2.7 to 10.2, $\mathrm{p}<0.001)$. Our subgroup analysis of the association between clot density and angiographic outcomes including only case series which reported the use of modern techniques also demonstrated that patients with a favorable angiographic outcome had a significantly higher mean clot HU than those with a poor angiographic outcome (MSD 6.7, 95\% CI 1.7 to $11.8, \mathrm{p}=0.009)$. $\mathrm{I}^{2}$ values for these estimates were $>50 \%$, indicating substantial heterogeneity.

\section{Imaging characteristics of thrombi and stroke etiology}

Seven articles with 738 patients examined the association between thrombi imaging characteristics and stroke etiology. 42328313334 Six studies examined CT findings and two studies examined MRI findings. Two CT studies $(33.3 \%, 2 / 6)$ found an association between the HAS and a cardioembolic etiology and four studies $(66.6 \%, 4 / 6)$ found no association. Both MRI studies found a positive association between the SVS and cardioembolic etiology.

Four studies had data available for meta-analysis examining the association between etiology and HAS. There was no association between imaging characteristics and stroke etiology (OR $1.13,95 \%$ CI 0.32 to $4.00, p=0.85)$. $\mathrm{I}^{2}$ values for this estimate were $>50 \%$, indicating substantial heterogeneity.

\section{DISCUSSION}

Our systematic review and meta-analysis of imaging and histopathologic characteristics of thrombi in acute ischemic stroke secondary to large vessel occlusion demonstrated a number of relevant findings. First, as the literature currently stands, the

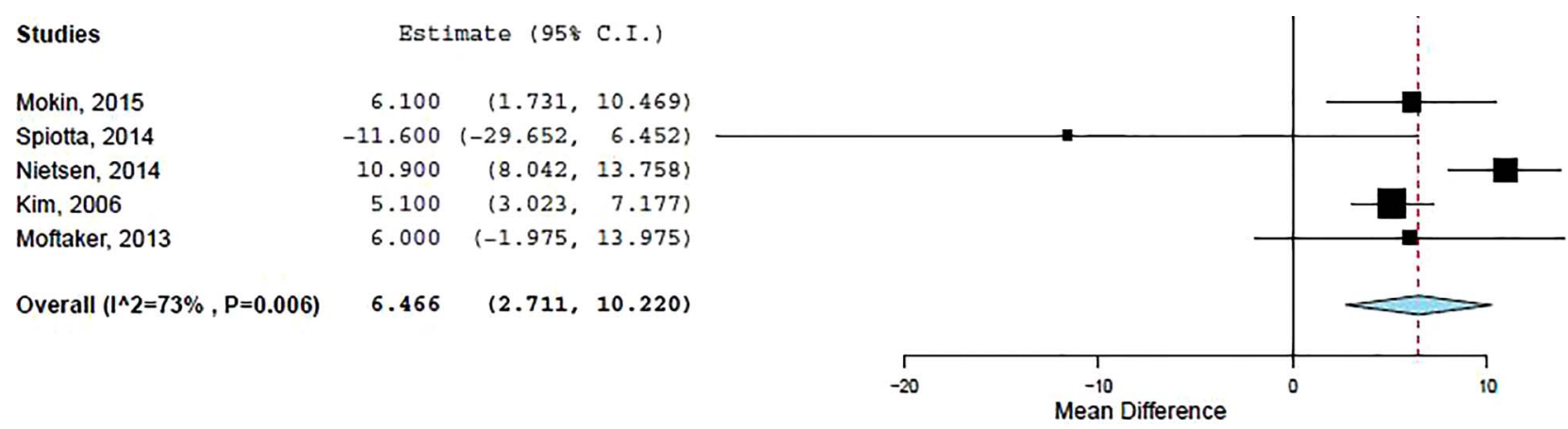

Figure 2 Forest plot comparing mean attenuation of clots in patients with good angiographic outcome versus those with poor angiographic outcome. Mean difference calculated as difference in the mean attenuation of patients with good angiographic outcome subtracted from the mean attenuation of patients with poor angiographic outcome. The positive value of the mean difference indicates that patients with a good angiographic outcome had a higher clot attenuation than those with a poor outcome. Good angiographic outcomes were defined as Thrombolysis In Myocardial Infarction (TIMI) score of $\geq 2$ or Thrombolysis In Cerebral Infarction (TICl) score of $\geq 2 \mathrm{~b}$. 
current characteristics of thrombi as determined by conventional staining techniques (ie, H\&E) do not reveal any information on stroke etiology. In addition, there appear to be only a few studies examining the association between the histologic composition of thrombi and revascularization outcomes. We found that patients with HAS had a higher odds of having RBC-rich thrombi than those without a HAS on CT (OR 9.0, 95\% CI 2.6 to $31.2, \mathrm{p}<0.01)$. Furthermore, the increasing vessel density on non-contrast CT was associated with increased recanalization with both IV-tPA and IA recanalization. Lastly, we found no association between thrombi imaging characteristics, including vessel density on CT and susceptibility on MRI, and stroke etiology. These findings suggest that, while imaging characteristics of thrombi in acute stroke can tell us important information regarding composition and potential revascularization outcomes, many important questions regarding the histopathologic characteristics of thrombi in acute ischemic stroke remain unanswered.

Understanding the histologic characteristics of thrombi in acute ischemic stroke is important for a number of reasons. First, in many cases, patients with acute ischemic stroke present with risk factors for both a large artery atherosclerotic and cardioembolic etiology for their ischemic event. While in many cases the etiology can be sorted out with a high degree of reliability, in cases of cryptogenic stroke-which comprise up to $30 \%$ of strokes depending on the population-the exact etiology remains uncertain. ${ }^{35}$ With improved understanding of the association between thrombi composition and etiology, thrombi composition could potentially provide an important clue regarding stroke etiology and thus affect secondary stroke prevention strategies. Half of the included studies in our systematic review found no association between thrombus composition and etiology while the remaining studies all yielded variable results. ${ }^{5}$ 12-19

All of the included studies used H\&E staining to evaluate thrombi composition. ${ }^{512-19}$ Based on these results, it is clear that $\mathrm{H} \& \mathrm{E}$ staining characteristics do not provide enough information regarding stroke etiology and further research examining cell subtypes using immunostaining methods are likely required to further understand the association between stroke etiology and thrombi composition. For example, some prior studies have suggested that vulnerable carotid plaques have high percentage areas of CD3-positive T cells and CD68- and CD11c-positive macrophages. ${ }^{36} 37$ With improved immunostaining methods it may be possible to create a profile of thrombi by their origins.

An understanding of the histologic characteristics of thrombi and recanalization outcomes is also potentially important in improving device selection and device development. ${ }^{3}$ 6-8 When engaging a thrombus with a stent-retriever or suction catheter, optimal outcomes are likely best achieved when the entirety of the thrombus is able to be retrieved in one pass. Multiple passes probably indicate that the thrombus is adherent to the vessel or fragmented, which could put the patient at risk for distal emboli and less than optimal recanalization outcomes. ${ }^{3}$ Furthermore, it is possible that the mechanical characteristics of a thrombus (soft vs stiff) affect the ability of a stent-retriever device or suction catheter to engage it. ${ }^{26-8}$ By understanding the distribution of thrombi phenotypes and their association with recanalization outcomes, we can arrive at a better understanding of why it is that successful recanalization is only achieved in $70-80 \%$ of cases and develop technologies that can be used to successfully retrieve these difficult thrombi. Our review found only three studies which examined the association between thrombi composition and outcome. Thus, it is clear that further research in this field is needed.

Ultimately, if histopathological studies of thrombi composition prove to be successful in demonstrating their association with etiology and recanalization outcomes, the importance of thrombi imaging will only increase. Based on findings from this review, it is clear that a HAS or SVS is a marker of an RBC-rich thrombus while the absence of these imaging findings is associated with a more fibrin-rich thrombus. ${ }^{4-6} 811$ 14-16 If, indeed, thrombus composition is shown to be associated with improved outcomes with certain techniques or devices, or if certain thrombi are shown to be associated with higher rates of fragmentation and distal embolization, imaging can then be used to guide device selection and at least provide the operator with a sense of what to expect during the revascularization procedure (ie, stiffness of thrombi or device/clot interaction). However, further understanding of the association between imaging characteristics and etiology could be useful to help determine stroke etiology in cases in which revascularization was either not performed or unsuccessful.

\section{Study limitations}

Our study has substantial limitations. First, there are relatively few studies in the literature examining many of the outcomes included in this study. A major finding of this analysis is the fact that much work remains to be done in examining the histologic and imaging characteristics of thrombi in acute stroke. In general, there was a lack of uniformity in the histopathologic descriptions of retrieved thrombi. Definitions included old versus fresh, RBC-rich versus fibrin-rich, serpentine versus laminated, and WBC-rich versus WBC-poor. Given the lack of standard nomenclature in thrombi characterization, this limits the generalizability of our results. It is also important to point out that some of the studies included in this analysis were performed prior to the advent of stent-retrievers and aspiration thrombectomy; technologies which now allow us to extract clots in their entirety rather than in fragments. This might affect sampling of clot histology. Stroke etiology was generally evaluated by a neurologist in the series that reported it. However, the details of this investigation were not always included in the included studies. Imaging techniques were variable across studies and imaging outcomes were generally not evaluated by an independent core laboratory.

There is inherent bias in assessing histologic characteristics of thrombi and angiographic outcomes as patients with poor outcomes presumably have no thrombi retrieved for analysis. There was a lack of uniformity in how many outcomes were reported, as some studies characterized imaging and pathological characteristics of thrombi using categorical variables while others used continuous variables. Most of the studies included in this systematic review and meta-analysis were retrospective single-center studies and at risk of selection bias. Nevertheless, this systematic review and meta-analysis provides important information regarding the current state of the literature on thrombi imaging and histology which can be further built upon in future studies.

\section{CONCLUSION}

Our systematic review and meta-analysis of thrombi imaging and histologic characteristics demonstrated a number of interesting findings. First, imaging characteristics including the HAS and SVS are associated with RBC-rich thrombi and HAS is associated with improved recanalization rates. Second, there were no definitive associations between clot histology using $\mathrm{H} \& \mathrm{E}$ 
staining and clot origin. Clearly, much work remains to be done in understanding the association between thrombi composition and stroke etiology as well as revascularization outcomes. Further research in the field of thrombi research in acute ischemic stroke is needed to address these questions and should focus on determining if certain device classes are better suited to treat different thrombi types, the role of immunostaining and other histologic classification techniques, and whether preoperative imaging can be used to guide device selection and revascularization techniques.

\section{Author affiliations \\ ${ }^{1}$ Department of Radiology, Mayo Clinic, Rochester, Minnesota, USA \\ ${ }^{2}$ Department of Engineering, Galway-Mayo Institute of Technology, Galway, Ireland \\ ${ }^{3}$ Department of Neurology, University of Heidelberg, Heidelberg, Germany \\ ${ }^{4}$ Department of Neurology, University of California, Los Angeles, California, USA \\ ${ }^{5}$ Department of Neurology, Erasmus MC University Medical Center, Rotterdam, The Netherlands \\ ${ }^{6}$ Department of Radiology, Academic Medical Center, Amsterdam, The Netherlands \\ ${ }^{7}$ Department of Neurosurgery, University of Buffalo, Buffalo, New York, USA \\ ${ }^{8}$ Department of Neurology, University of Cincinatti, Cincinatti, Ohio, USA \\ ${ }^{9}$ Department of Radiology, University of Tennessee Medical Center, Chatanooga, \\ Tennessee, USA \\ ${ }^{10}$ Department of Neurology, Emory University, Atlanta, Georgia, USA \\ ${ }^{11}$ Department of Radiology, New England Center for Stroke Research, University of \\ Massachusetts, Worcester, Massachusetts, USA \\ ${ }^{12}$ Department of Neurology, University of Pittsburgh Medical Center, Pittsburgh, Pennsylvania, USA}

Contributors All authors participated in drafting the article and revising it critically for important intellectual content. These authors made substantial contributions to conception and design, acquisition of data, and analysis and interpretation of data. All authors provided final approval of the version to be published. Waleed Brinjikji M.D. and Sharon Duffy contributed equally to this work.

Competing interests None declared.

Provenance and peer review Not commissioned; internally peer reviewed.

Data sharing statement Please contact the corresponding author for data sharing requests.

\section{REFERENCES}

1 Ahn SH, Choo IS, Hong R, et al. Hyperdense arterial sign reflects the proportion of red blood cells in the thromboemboli of acute stroke patients. Cerebrovasc Dis 2012:33:236.

2 Chueh JY, Wakhloo AK, Hendricks GH, et al. Mechanical characterization of thromboemboli in acute ischemic stroke and laboratory embolus analogs. AJNR Am J Neuroradiol 2011;32:1237-44.

3 Froehler MT, Tateshima S, Duckwiler G, et al. The hyperdense vessel sign on CT predicts successful recanalization with the Merci device in acute ischemic stroke. J Neurointerv Surg 2013;5:289-93.

4 Guthrie S, Huang X, Moreton F, et al. The significance of the hyperdense vessel sign (HVS). Int I Stroke 2012;7(Suppl 2):3.

5 Liebeskind DS, Sanossian N, Yong WH, et al. CT and MRI early vessel signs reflect clot composition in acute stroke. Stroke 2011;42:1237-43.

6 Mehta BP, Nogueira RG. Should clot composition affect choice of endovascular therapy? Neurology 2012;79(Suppl 1):S63-7.

7 van der Marel K, Chueh JY, Brooks OW, et al. Quantitative assessment of device-clot interaction for stent retriever thrombectomy. J Neurointerv Surg. Published Online First: 1 Feb 2016.

8 Nogueira RG, Levy El, Gounis M, et al. The Trevo device: preclinical data of a nove stroke thrombectomy device in two different animal models of arterial thrombo-occlusive disease. J Neurointerv Surg 2012;4:295-300.

9 DerSimonian R, Laird N. Meta-analysis in clinical trials. Control Clin Trials 1986;7:177-88.

10 Higgins JP, Thompson SG, Deeks JJ, et al. Measuring inconsistency in meta-analyses. BMJ 2003;327:557-60.

11 Deeks J, Dinnes J, D'Amico R, et al. Evaluating non-randomised intervention studies. Health Technol Assess 2003;7:iii-x, 1-173.

12 Boeckh-Behrens T, Schubert M, Förschler A, et al. The impact of histological clot composition in embolic stroke. Clin Neuroradiol. Published Online First: 27 Sep 2014.
13 Kim SK, Yoon W, Kim TS, et al. Histologic analysis of retrieved clots in acute ischemic stroke: correlation with stroke etiology and gradient-echo MRI. AJNR Am J Neuroradiol 2015;36:1756-62.

14 Marder VJ, Chute DJ, Starkman S, et al. Analysis of thrombi retrieved from cerebral arteries of patients with acute ischemic stroke. Stroke 2006;37:2086-93.

15 Niesten JM, van der Schaaf IC, van Dam L, et al. Histopathologic composition of cerebral thrombi of acute stroke patients is correlated with stroke subtype and thrombus attenuation. PLOS ONE 2014;9:e88882.

16 Sallustio F, Koch G, Di Legge $S$, et al. Histological features of thrombo-embolic specimens collected from intracranial arteries of patients with acute ischemic stroke. Cerebrovasc Dis 2013;35:180.

17 Sato $\mathrm{Y}$, Ishibashi-Ueda $\mathrm{H}$, Iwakiri T, et al. Thrombus components in cardioembolic and atherothrombotic strokes. Thromb Res 2012;130:278-80.

18 Simons N, Mitchell P, Dowling R, et al. Thrombus composition in acute ischemic stroke: a histopathological study of thrombus extracted by endovascular retrieval. J Neuroradiol 2015;42:86-92.

19 Singh P, Doostkam S, Reinhard M, et al. Cerebrovascular occlusive disease histopathological analysis of thrombi retrieved by endovascular mechanical extraction during acute ischemic stroke. Int J 2014;9:143.

20 Qureshi MH, Lobanova I, Suri FK, et al. Histopathological characteristics of IV recombinant tissue plasminogen resistant thrombi in patients with acute ischemic stroke [abstract]. Stroke 2015;46.

21 Cline B, Vos J, Carpenter J, et al. Pathological analysis of extracted clots in embolectomy patients with acute ischaemic stroke [abstract]. J Neurointerv Surg 2013;5:A15-16.

22 Lopez-Cancio E, Millan M, Perez de la Ossa N, et al. Immunohistochemical study of clot composition in thrombi retrieved from MCA with mechanical thrombectomy. Cerebrovasc Dis 2013;35:255.

23 Kim EY, Heo JH, Lee SK, et al. Prediction of thrombolytic efficacy in acute ischemic stroke using thin-section noncontrast CT. Neurology 2006;67:1846-8.

24 Kolieskova S, Bag AK, Barlinn K, et al. Hyperdense middle cerebral artery hounsfield units predict early recanalization in patients with acute ischemic stroke. Cerebrovasc Dis 2012;33:234-5.

25 Moftakhar P, English JD, Cooke DL, et al. Density of thrombus on admission CT predicts revascularization efficacy in large vessel occlusion acute ischemic stroke. Stroke 2013;44:243-5.

26 Mokin M, Morr S, Natarajan SK, et al. Thrombus density predicts successful recanalization with Solitaire stent retriever thrombectomy in acute ischemic stroke. J Neurointerv Surg 2015;7:104-7.

27 Niesten JM, van der Schaaf IC, van der Graaf Y, et al. Predictive value of thrombus attenuation on thin-slice non-contrast $\mathrm{CT}$ for persistent occlusion after intravenous thrombolysis. Cerebrovasc Dis 2014;37:116-22.

28 Puig J, Pedraza S, Demchuk A, et al. Quantification of thrombus hounsfield units on noncontrast $\mathrm{CT}$ predicts stroke subtype and early recanalization after intravenous recombinant tissue plasminogen activator. AJNR Am J Neuroradiol 2012; 33:90-6.

29 Schellinger PD, Chalela JA, Kang DW, et al. Diagnostic and prognostic value of early MR imaging vessel signs in hyperacute stroke patients imaged $<3$ hours and treated with recombinant tissue plasminogen activator. AJNR Am I Neuroradiol 2005:26:618-24.

30 Soize S, Batista AL, Rodriguez Regent C, et al. Susceptibility vessel sign on T2* magnetic resonance imaging and recanalization results of mechanical thrombectomy with stent retrievers: a multicentre cohort study. Eur I Neurol 2015;22:967-72.

31 Topcuoglu MA, Arsava EM, Kursun 0, et al. The utility of middle cerebral artery clot density and burden assessment by noncontrast computed tomography in acute ischemic stroke patients treated with thrombolysis. J Stroke Cerebrovasc Dis 2014;23:e85-91.

32 Yan S, Liu K, Tong L, et al. Different risk factors for poor outcome between patients with positive and negative susceptibility vessel sign. J Neurointerv Surg. Published Online First: 25 Sep 2015.

33 Almekhlafi MA, Menon B, Goyal M, et al. Role of biological markers in predicting findings of multimodal thrombus imaging: Implications for aetiology and recanalization. Stroke 2011;42:e599.

34 Cho KH, Kim JS, Kwon SU, et al. Significance of susceptibility vessel sign on T2*-weighted gradient echo imaging for identification of stroke subtypes. Stroke 2005;36:2379-83.

35 Goldstein LB, Jones MR, Matchar DB, et al. Improving the reliability of stroke subgroup classification using the Trial of ORG 10172 in Acute Stroke Treatment (TOAST) criteria. Stroke 2001;32:1091-8.

36 Shaikh $S$, Brittenden J, Lahiri $R$, et al. Macrophage subtypes in symptomatic carotid artery and femoral artery plaques. Eur J Vasc Endovasc Surg 2012;44:491-7.

37 Cho KY, Miyoshi H, Kuroda S, et al. The phenotype of infiltrating macrophages influences arteriosclerotic plaque vulnerability in the carotid artery. I Stroke Cerebrovasc Dis 2013;22:910-18. 